1999-6

\title{
Synthesis and Experimental Testing of a Nonlinear Optimal Tracking Controller
}

Timothy McLain

Mechanical Engineering Department, Brigham Young University, mclain@byu.edu

Randal W. Beard

Department of Electrical and Computer Engineering, Brigham Young University, beard@ee.byu.edu

Christopher A. Bailey

Mechanical Engineering Department, Brigham Young University

Follow this and additional works at: https://scholarsarchive.byu.edu/facpub

Part of the Mechanical Engineering Commons

\section{Original Publication Citation}

McLain, T., Bailey, C., and Beard, R. Synthesis and Experimental Testing of a Nonlinear Optimal Tracking Controller, Proceedings of the American Control Conference, vol. 4, pp. 2847-2851, June 1999, San Diego, California.

\section{BYU ScholarsArchive Citation}

McLain, Timothy; Beard, Randal W.; and Bailey, Christopher A., "Synthesis and Experimental Testing of a Nonlinear Optimal Tracking Controller" (1999). Faculty Publications. 1936.

https://scholarsarchive.byu.edu/facpub/1936 


\title{
Synthesis and Experimental Testing of a Nonlinear Optimal Tracking Controller
}

\author{
Timothy W. McLain ${ }^{1 *} \quad$ Christopher A. Bailey ${ }^{1} \quad$ Randal W. Beard ${ }^{2}$ \\ ${ }^{1}$ Department of Mechanical Engineering, Brigham Young University \\ ${ }^{2}$ Department of Electrical \& Computer Engineering, Brigham Young University
}

\begin{abstract}
This paper presents a novel approach for developing tracking controllers for nonlinear systems. The approach involves the numerical solution, by Galerkin approximation, of the time-varying Hamilton-Jacobi-Bellman (HJB) equation and results in a nonlinear controller approximating the optimal tracking control law for a specified desired trajectory and cost function. Experimental results are presented for a nonlinear pendulum system.
\end{abstract}

\section{Introduction}

Designing control laws that cause a nonlinear system to track a desired trajectory is a problem of fundamental importance in control theory. Standard approaches feedback linearization [8], and plant inversion [5]. Optimal control techniques can be formulated for nonlinear tracking, but the resulting control laws are typically open-loop (cf. [1, 9]). The difficulty is that the feedback problem reduces to solving the time-varying HJB equation which is difficult to solve analytically. In this paper we present an approach to approximating the timevarying HJB equation, and hence to designing tracking controllers for nonlinear systems. Our approach is to use the standard Galerkin approximation scheme [6] with all of the basis functions defined on a compact subset of the state space. Other approaches to approximating the time-varying HJB equation have appeared in the literature. In [7], the HJB equation is approximated by discretizing the time and spatial variables and solving the resulting finite-difference equations. In [12] the finitetime optimal control problem is solved by regularizing the cost function and solving the resulting Riccati differential equation. Perhaps the closest approach to ours is [13], where the time-varying HJB equation is approximated via a power series. Galerkin approximations of the time-varying HJB equation were first reported in [2]. The regulator problem has been treated in detail and results have appeared in $[3,4,10,11]$.

*Corresponding Author: email tmclain@et.byu.edu

\section{Control Approach}

Given a system modeled by the nonlinear state equations

$$
\dot{x}=f(x)+g(x) u(x, t)
$$

and the desired state trajectory $x_{d}(t)$, a finite-time performance index $V(x, t)$ can be formulated that will cause the states to track the desired trajectory

$$
\begin{aligned}
V(x, t) & =\int_{0}^{T}\left(l(x, t)+\|u(x, t)\|_{R}^{2}\right) d t+s[x(T)] \\
l(x, t) & =\left(x-x_{d}\right)^{T} Q\left(x-x_{d}\right) \\
s[x(T)] & =\left[x(T)-x_{d}(T)\right]^{T} S\left[x(T)-x_{d}(T)\right],
\end{aligned}
$$

where $s[x(T)]$ is the cost on the terminal error.

The optimal control which minimizes the performance index is given by

$$
u^{*}(x, t)=-\frac{1}{2} R^{-1} g^{T}(x) \frac{\partial V^{*}}{\partial x}
$$

where $V^{*}(x, t)$ is a positive definite function that satisfies the Hamilton-Jacobi-Bellman (HJB) optimization equation:

$$
\begin{aligned}
\frac{\partial V^{*}}{\partial t}+\frac{\partial V^{* T}}{\partial x} & f(x)+l(x, t) \\
& -\frac{1}{4} \frac{\partial V^{* T}}{\partial x} g(x) R^{-1} g^{T}(x) \frac{\partial V^{*}}{\partial x}=0 .
\end{aligned}
$$

Obtaining an analytical solution to Equation (4) for nonlinear physical systems of practical interest is typically not possible. The approach for solving the HJB partial differential equation presented here is numerical solution by Galerkin approximation. Using this approach, an accurate approximation to the optimal control solution can be obtained.

\subsection{Numerical Solution of the HJB Equa- tion}

To solve the HJB optimization equation, a computational Galerkin method is employed [6]. It is first assumed that $V$ can be written as an infinite series of known 
basis functions $\phi_{j}(x)$ that are continuous and defined on the closed and bounded set $\Omega$, the region of state space of specific interest, i.e.,

$$
V(x, t)=\sum_{j=1}^{\infty} c_{j}(t) \phi_{j}(x) \quad x \in \Omega .
$$

An approximation to the assumed solution $V$ having the desired degree of accuracy can be formed by considering the first $N$ terms of the infinite series:

$$
V_{N}(x, t)=\sum_{j=1}^{N} c_{j}(t) \phi_{j}(x) .
$$

By substituting Equation (5) into Equation (4), an expression for the error, $e$, resulting from the series approximation is obtained:

$$
\begin{aligned}
e(x, t) & =\sum_{j=1}^{N} \frac{d c_{j}}{d t} \phi_{j}+\sum_{j=1}^{N} c_{j} \frac{\partial \phi_{j}^{T}}{\partial x} f(x)+l(x, t) \\
& -\frac{1}{4} \sum_{j=1}^{N} c_{j} \frac{\partial \phi_{j}^{T}}{\partial x} g(x) R^{-1} g^{T}(x) \sum_{j=1}^{N} c_{j} \frac{\partial \phi_{j}}{\partial x} .
\end{aligned}
$$

By Galerkin's method, the unknown coefficients $\left\{c_{i}\right\}_{1}^{N}$ can be found by projecting the error equation onto the $N$ basis functions $\phi_{1}, \phi_{2}, \ldots, \phi_{N}$ on the domain $\Omega$ and setting them to zero. The projection of $e(x, t)$ onto $\{\phi\}_{1}^{N}$ is found by taking the inner product of $e(x, t)$ and $\{\phi\}_{1}^{N}$, where the inner product of two functions $a$ on $b$ is defined in the following manner:

$$
\langle a(x), b(x)\rangle_{\Omega} \triangleq \int_{\Omega} a(x) b(x) d x .
$$

By projecting the error onto the basis functions, $N$ nonlinear ordinary differential equations in the $N$ unknown coefficients $\left(c_{1}(t), c_{2}(t), \ldots, c_{N}(t)\right)$ are obtained:

$$
\left\langle e(x, t), \phi_{j}(x)\right\rangle_{\Omega}=0, \quad j=1 \ldots N .
$$

Equation (7) can be expressed in matrix form as

$$
A_{1} \dot{c}+A_{2} c+b_{1}+b_{2}=0
$$

with final condition

$$
c(T)=A_{1}^{-1}\left[\begin{array}{c}
\left\langle s[x(T)], \phi_{1}\right\rangle_{\Omega} \\
\vdots \\
\left\langle s[x(T)], \phi_{N}\right\rangle_{\Omega}
\end{array}\right]
$$

where

$$
\begin{aligned}
A_{1}= & {\left[\begin{array}{ccc}
\left\langle\phi_{1}, \phi_{1}\right\rangle_{\Omega} & \cdots & \left\langle\phi_{N}, \phi_{1}\right\rangle_{\Omega} \\
\vdots & \ddots & \vdots \\
\left\langle\phi_{1}, \phi_{N}\right\rangle_{\Omega} & \cdots & \left\langle\phi_{N}, \phi_{N}\right\rangle_{\Omega}
\end{array}\right] } \\
A_{2}= & {\left[\begin{array}{ccc}
\left\langle\frac{\partial \phi_{1}^{T}}{\partial x} f, \phi_{1}\right\rangle_{\Omega} & \cdots & \left\langle\frac{\partial \phi_{N}^{T}}{\partial x} f, \phi_{1}\right\rangle_{\Omega} \\
\vdots & \ddots & \vdots \\
\left\langle\frac{\partial \phi_{1}^{T}}{\partial x} f, \phi_{N}\right\rangle_{\Omega} & \cdots & \left\langle\frac{\partial \phi_{N}^{T}}{\partial x} f, \phi_{N}\right\rangle_{\Omega}
\end{array}\right] }
\end{aligned}
$$

$$
\begin{aligned}
& b_{1}=-\left[\begin{array}{c}
\left\langle l(x, t), \phi_{1}\right\rangle_{\Omega} \\
\vdots \\
\left\langle l(x, t), \phi_{N}\right\rangle_{\Omega}
\end{array}\right] \\
& b_{2}=-\frac{1}{4}\left[\begin{array}{c}
c^{T} M_{1} c \\
c^{T} M_{2} c \\
\vdots \\
c^{T} M_{N} c
\end{array}\right] \\
&\left\{M_{i}\right\}_{1}^{N}= {\left[\begin{array}{ccc}
m_{i}(1,1) & \ldots & m_{i}(1, N) \\
\vdots & \ddots & \vdots \\
m_{i}(N, 1) & \ldots & m_{i}(N, N)
\end{array}\right] } \\
& m_{i}(j, k)=\left\langle\frac{\partial \phi_{k}^{T}}{\partial x} g R^{-1} g^{T} \frac{\partial \phi_{i}}{\partial x}, \phi_{j}\right\rangle_{\Omega} .
\end{aligned}
$$

This system of ordinary differential equations can be solved numerically to find $c(t)=\left[\begin{array}{llll}c_{1} & c_{2} & \cdots & c_{N}\end{array}\right]^{T}$. With these coefficients identified, the functional expression for the approximate optimal tracking control law can be calculated:

$$
\begin{aligned}
u_{N}(x, t) & =-\frac{1}{2} R^{-1} g^{T}(x) \frac{\partial V_{N}}{\partial x} \\
& =-\frac{1}{2} R^{-1} g^{T}(x)\left(\sum_{j=1}^{N} c_{j}(t) \frac{\partial \phi_{j}}{\partial x}\right)
\end{aligned}
$$

\section{Nonlinear Pendulum System}

As an example application of the nonlinear optimal tracking approach developed above, a controller will be developed for an actuated pendulum system moving in the vertical plane shown in Figure 1. The dynamics of the system are described by the following equations of motion

$$
\begin{gathered}
\dot{\omega}=-\frac{b}{J} \omega-\frac{T_{c}}{J} \operatorname{sign}(\omega)+\frac{m g L}{J} \sin \theta+\frac{K_{\tau}}{J} i(t) \\
\dot{\theta}=\omega,
\end{gathered}
$$

where $J$ is the moment of inertia of the rod, $m$ is the mass of the rod, $b$ is the viscous friction coefficient, $T_{c}$ is the coulomb friction torque level, and $L$ is the distance from the pendulum pivot to the pendulum center of mass. The nonlinear elements of the system are the gravitational moment and the coulomb friction. Equations (10) and (11) are of the general form of Equation (1) and from them the functions $f(x)$ and $g(x)$ required for the synthesis of the control can be determined.

\section{Control Synthesis and Implementation}

With the model described above, the optimal feedback control can be synthesized based on Equation (8). With 


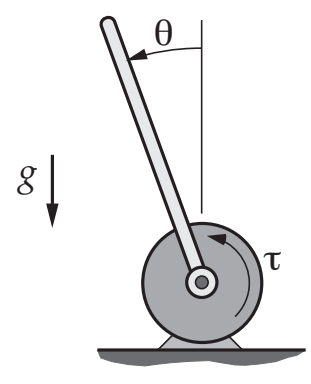

Figure 1: Pendulum System

$f(x)$ and $g(x)$ coming directly from the equations of motion, the cost function on the states $l(x, t)$, the weighting matrix on the control cost $R$, the weighting matrix on the terminal error $S$, the domain of the states $\Omega$, and the basis functions $\left\{\phi_{j}(x)\right\}_{1}^{N}$ remain to be determined. For the results presented here, the cost function was chosen to be

$$
l(x, t)=q_{1}\left(\omega-\omega_{d}\right)^{2}+q_{2}\left(\theta-\theta_{d}\right)^{2} .
$$

Since the system has only a single input $\left(i_{v}\right)$, the weighting matrix on the control $(R)$ is simply a scalar variable. The weighting matrix on the terminal error, $S$, was set to be a zero matrix. The domain of possible values for the states is governed by the physical limitations of the system and the trajectories being tracked. In this case, the domain of the states was chosen to be

$$
\begin{gathered}
-10 \mathrm{rad} / \mathrm{s} \leq \omega \leq 10 \mathrm{rad} / \mathrm{s} \\
-\pi \mathrm{rad} \leq \theta \leq \pi \mathrm{rad} .
\end{gathered}
$$

Selection of the basis functions $\left\{\phi_{j}(x)\right\}_{1}^{N}$ is an important part of the design process. Not only are the basis functions used to approximate the cost function, they also determine the form of the control law. In an effort to compensate adequately for the nonlinear dynamics of the system, the following basis functions were used for this problem:

$$
\begin{aligned}
\left\{\phi_{j}\right\}_{j=1}^{10}=\left\{\omega^{2}, \omega \theta, \theta^{2}, \omega, \theta,\right. & \sin \theta, \cos \theta, \\
\omega & \left.\sin \theta, \theta \sin \theta, \sin ^{2} \theta\right\} .
\end{aligned}
$$

Finally, the desired state trajectories were chosen to be 5th-order spline polynomials between initial and final states. Trajectories that can be specified as functions of time are most easily implemented using this approach.

With the required parameters defined, coefficient trajectories $c_{1}(t), c_{2}(t), \ldots, c_{11}(t)$ that minimize the cost function of Equation (3) for a specified trajectory can be found by numerically solving the ordinary differential equation (8). From these coefficient trajectories, the optimal feedback control law can be found from Equation (9). When implemented in hardware, the coefficient trajectories are stored in a lookup table and accessed accordingly during the execution of the trajectory.
Table 1: Weighting Functions and Control Laws

\begin{tabular}{|l|}
\hline Initial Design \\
\hline$l=\left(\omega_{d}-\omega\right)^{2}+2.5\left(\theta_{d}-\theta\right)^{2}$ \\
$R=1$ \\
$u=-4.154\left[2 c_{1}(t) \omega+c_{2}(t) \theta+c_{4}(t)+c_{8}(t) \sin \theta\right]$ \\
\hline \hline High-Performance Design \\
\hline$l=10\left(\omega_{d}-\omega\right)^{2}+100\left(\theta_{d}-\theta\right)^{2}$ \\
$R=1$ \\
$u=-4.154\left[2 c_{1}(t) \omega+c_{2}(t) \theta+c_{4}(t)+c_{8}(t) \sin \theta\right]$ \\
\hline \hline Moderate-Control Design \\
\hline$l=10\left(\omega_{d}-\omega\right)^{2}+100\left(\theta_{d}-\theta\right)^{2}$ \\
$R=5$ \\
$u=-0.8307\left[2 c_{1}(t) \omega+c_{2}(t) \theta+c_{4}(t)+c_{8}(t) \sin \theta\right]$ \\
\hline
\end{tabular}

\section{$5 \quad$ Results}

Using the synthesis approach outlined above, three different optimal-tracking controllers were designed. Table 1 shows the weighting functions and resulting control laws for each of the controllers presented. Control laws were calculated according to Equation (9).

Though there are ten coefficient trajectories generated from the solution of Equation (8), only $c_{1}(t), c_{2}(t), c_{4}(t)$, and $c_{8}(t)$ contribute explicitly to the final control law. This is due to the zero terms present in the $g(x)$ vector and the $\frac{\partial \phi}{\partial x}$ matrix. Even though the remaining coefficient trajectories are not present in the control law expression, they are coupled through the ordinary differential equations describing the coefficients (Equation (8)) and hence influence the control law indirectly.

As an example of the coefficient profiles $c_{j}(t)$ obtained from the control law synthesis process, profiles for the high-performance design of Table 1 are presented in Figure 2 . The coefficient profiles contain information about both the nonlinear dynamics of the system and the trajectory for which the control law was designed. In this specific example, $c_{4}(t)$ is a linear combination of the $\theta_{d}$ and $\omega_{d}$ trajectories. Thus, the time-varying coefficients enable trajectory tracking even though $\theta_{d}$ and $\omega_{d}$ are not present explicitly in the control law.

Figures 3 through 5 present results for the three different controller designs. For each controller, a design was synthesized for a 5th-order polynomial spline trajectory from $-\pi / 2$ to $\pi / 4$ radians having a duration of 0.7 seconds using the weighting functions of Table 1.

Figure 3 show the tracking response of the system when the weights on the trajectory errors and control are of similar magnitude. It can be seen that the tracking errors are significant, especially in the steady-state portion of the trajectory. For the given cost function, decreasing the steady-state error (with larger steady-state control) would result in an increase in the performance index. 

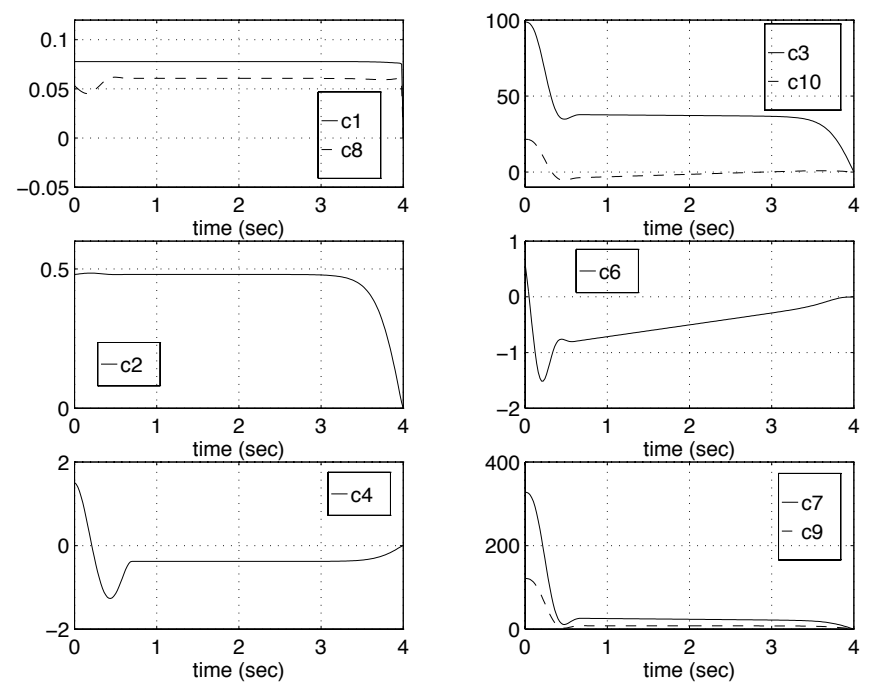

Figure 2: Coefficient Trajectories
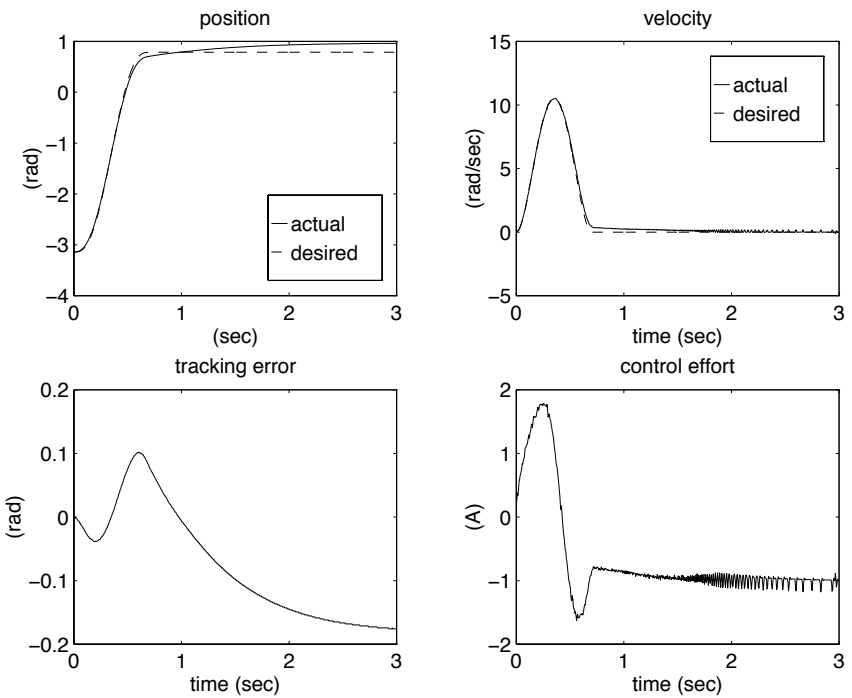

Figure 3: Tracking Response - Initial

Thus for the weights at these initial values, the "optimal" control results in significant steady-state tracking errors.

Tracking errors can be reduced by increasing the weights on the tracking errors. Figure 4 shows the highperformance tracking response obtained when the cost of tracking errors is very large relative to the cost of the control. As expected, the tracking errors are much smaller both during the transient and steady-state portions of the trajectory. Increasing the weights on the tracking errors caused the position error to be reduced dramatically with only slightly more control effort. Unlike a regulator, where a significant increase of the weights on state errors would increase the speed of the transient response, the speed of the transient response (and hence the amount of control effort expended) is governed largely by the spec-
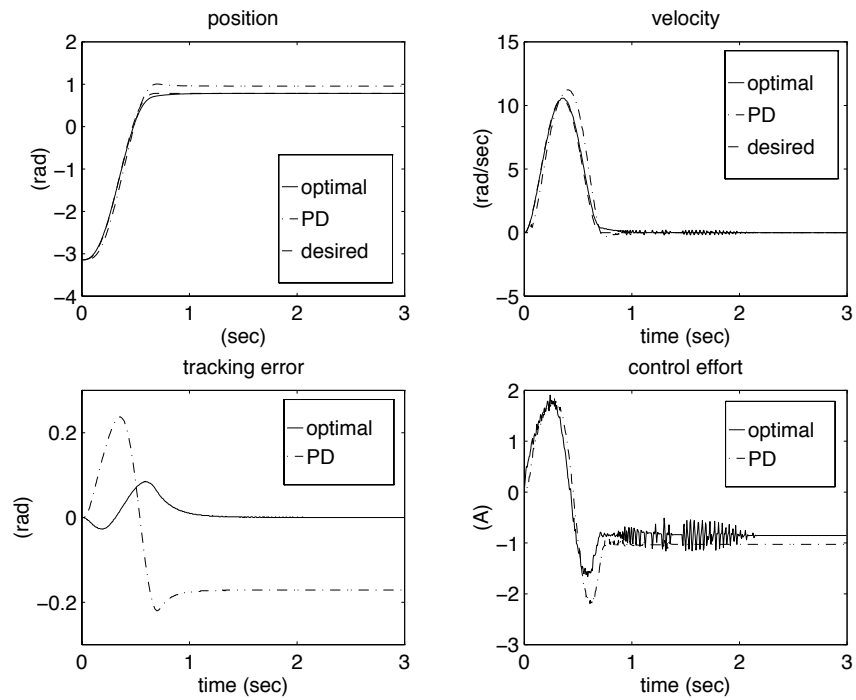

Figure 4: Tracking Response — High Performance
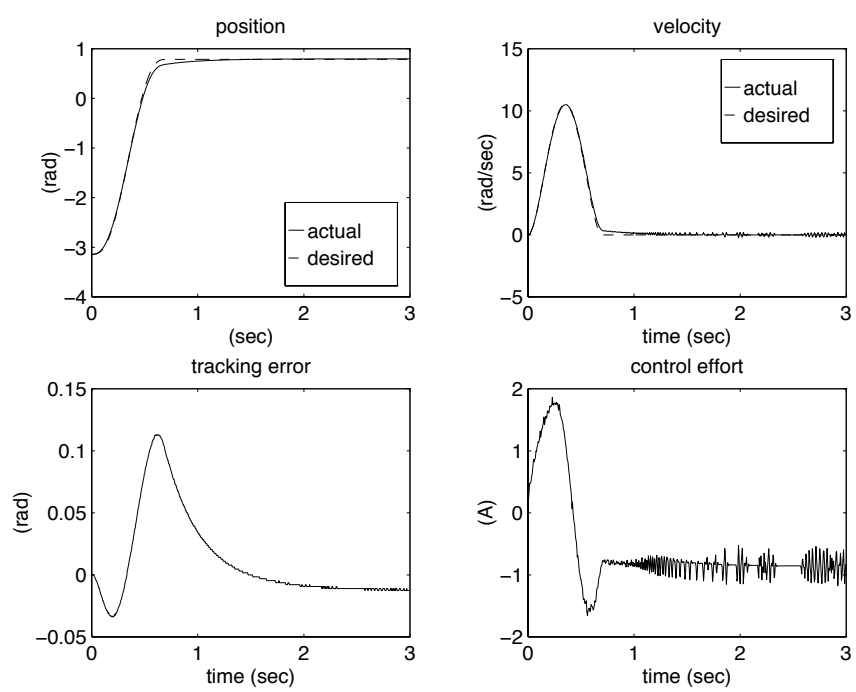

Figure 5: Tracking Response - Moderated

ified trajectory.

For comparison, the response of a well-tuned PD controller is shown in Figure 4 together with that of the optimal tracking controller. The primary difference between the optimal and PD controllers is the existence of the $c_{8} \sin \theta$ term in the optimal control law. The optimal synthesis results in a value of $c_{8}$ that counteracts almost exactly the gravitational load exerted on the pendulum, thereby reducing the error throughout the trajectory and especially in steady state. Due to this term, all of the optimal trajectory controllers tested exhibited improved performance over the PD control design.

Control effort usage can be moderated by increasing the weight on the control $R$. Figure 5 shows the response of the system with the controller having the same tracking-error weights as the high-performance design, 
but with the weight on the control increased by a factor of five. As expected, the tracking errors increase due to the increased cost of the control, while the control usage decreases slightly.
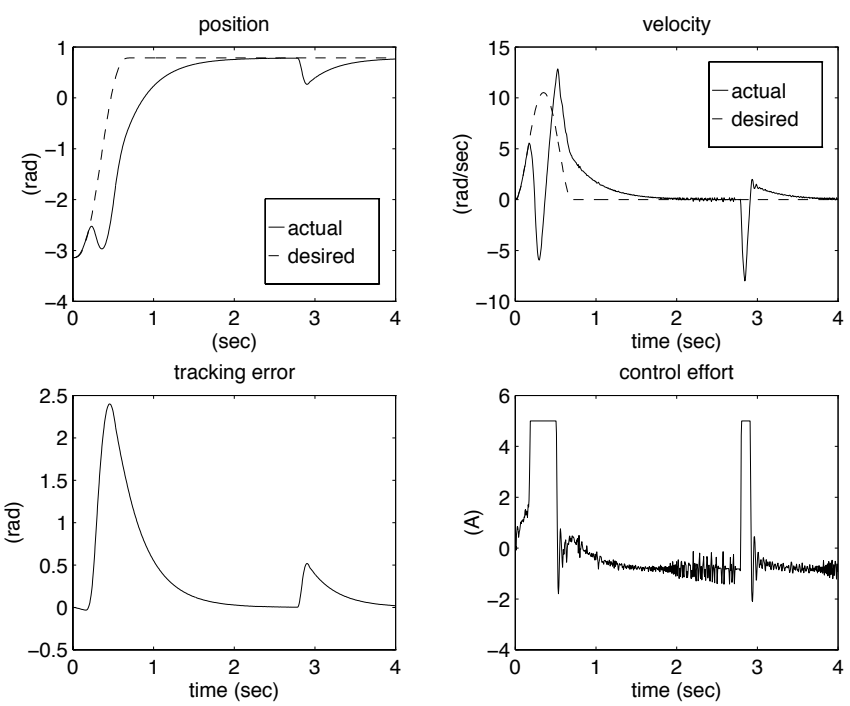

Figure 6: Disturbance Rejection

An unexpected (though pleasing) result of this design method is that it is robust to disturbances and modeling errors. With most feedback control designs, tracking error is evident explicitly in the control law, and the behavior of the control law which drives the tracking error to zero (even in the presence of disturbances) is intuitively obvious. With the method presented here, there are no tracking error terms in the control law causing one to question how the tracking error will be driven to zero. As discussed previously, information about the desired state trajectories is held in the coefficient trajectories and though not expressed explicitly, tracking error is embedded in the formulation of the control law. Figure 6 illustrates the disturbance rejection capabilities of the high-performance control design. In this demonstration, the system is given a torque disturbance midway through the trajectory and another after the completion of the trajectory. Control effort values above $5 \mathrm{~A}$ are clipped due to the limitations of the motor amplifier.

\section{Conclusions}

An approach for the design of optimal tracking controllers for nonlinear systems has been presented. This approach is based on the solution of the Hamilton-Jacobi partial differential equation using a numerical Galerkin strategy. The resulting optimal control laws exhibit a time-varying dependence on the specified trajectory and cost function and the dynamics of the system involved.

Using this synthesis strategy, several optimal tracking controllers were designed for a nonlinear pendulum system. Experimental results demonstrated the ability to alter the dynamics of the closed-loop control system by tuning weights on the trajectory error and the control effort. Robustness to external disturbances and modeling error were also demonstrated experimentally.

\section{References}

[1] Z. Aganovic And Z. GAJIC, The successive approximation procedure for finite-time optimal control of bilinear systems, IEEE Transactions on Automatic Control, 39 (1994), pp. 1932-1935.

[2] R. BEARD, Improving the Closed-Loop Performance of Nonlinear Systems, PhD thesis, Rensselaer Polytechnic Institute, Troy, New York, 1995.

[3] R. Beard, G. Saridis, and J. Wen, Improving the performance of stabilizing control for nonlinear systems, Control Systems Magazine, 16 (1996), pp. 27-35.

[4] R. W. Beard and T. W. McLain, Successive Galerkin approximation algorithms for nonlinear optimal and robust control, International Journal of Control: Special Issue on Breakthroughs in the Control of Nonlinear Systems, 71 (1998), pp. 717-743.

[5] S. Devasia, D. Chen, and B. Paden, Nonlinear inversion-based output tracking, IEEE Transactions on Automatic Control, 41 (1996), pp. 930-942.

[6] C. A. J. Fletcher, Computational Galerkin Methods, Springer Series in Computational Physics, Springer Verlag, New York, New York, 1984.

[7] R. Gonzalez and E. Rofman, On deterministic control problems: An approximation procedure for the optimal cost ii: The nonstationary problem, SIAM Journal on Control and Optimization, 23 (1985), pp. 267-285.

[8] I. J. Ha AND E. G. GILBERT, Robust tracking in nonlinear systems, IEEE Transactions on Automatic Control, 32 (1987), pp. 763-771.

[9] E. P. Hofer And B. Tibken, An iterative method for the finite-time bilinear-quadratic control problem, Journal of Optimization Theory and Applications, 57 (1988), pp. 411-427.

[10] T. W. McLain and R. W. Beard, Nonlinear optimal control of a hydraulically actuated positioning system, in Proceedings of the ASME International Mechanical Engineering Congress and Exposition, Fluid Power Systems and Technology Division, Dallas, TX, November 1997.

[11] _ Nonlinear optimal control design of a missile autopilot, in AIAA Guidance, Navigation and Control Conference, Boston, MA, 1998.

[12] E. P. RYan, Optimal feedback control of bilinear systems, Journal of Optimization Theory and Applications, 44 (1984), pp. 333-362.

[13] A. P. WiLLEMSTEIN, Optimal regulation of nonlinear $d y$ namical systems on a finite interval, SIAM Journal on Control and Optimization, 15 (1977), pp. 1050-1069. 